\title{
Rendimento na barra de tração de um trator agrícola com diferentes relações de peso e potência ${ }^{1}$
}

\author{
Tractor drawbar efficiency at different weight and power ratios
}

\author{
Leonardo de Almeida Monteiro ${ }^{2 *}$, Daniel Albiero ${ }^{2}$, Fabio Henrique de Souza ${ }^{2}$, Rafaela Paula Melo $^{2}$ e Igor \\ Martins Cordeiro ${ }^{2}$
}

\begin{abstract}
RESUMO - O ensaio do trator em solo agrícola é uma das maneiras de se obter informações, principalmente no que diz respeito ao seu desenvolvimento de tração. O rendimento na barra de tração é frequentemente usado para comparar ou avaliar tratores. Entretanto, o rendimento na barra de tração é afetado pelas condições da superfície de deslocamento, pela relação de engrenagens e pela relação entre o peso e a potência do trator. O objetivo do presente trabalho foi avaliar o rendimento na barra de tração de um trator agrícola com diferentes relações entre o peso/potência sob diferentes regimes de carga aplicados na barra de tração. O ensaio foi conduzido na Fazenda Experimental Lageado, pertencente a UNESP, Campus de Botucatu - SP avaliando as seguintes relações de peso e potência: $50 ; 55$ e $60 \mathrm{~N} \mathrm{~kW}^{-1}$, onde para cada uma dessas relações utilizou-se três intervalos de força na barra de tração da seguinte forma: 15a 20; 25 a 30 e 35 a $40 \mathrm{kN}$. Concluiuse que as relações de peso e potência e as cargas estudadas apresentaram pouca variabilidade. Observou-se que a relação de peso e potência de 50 e $55 \mathrm{~N} \mathrm{~kW}^{-1}$, para as cargas aplicadas na barra de tração de 25 a 30 e 35 a $40 \mathrm{kN}$, apresentaram diferença entre médias, sendo que o maior rendimento da barra de tração foi obtido para a carga de 25 a $30 \mathrm{kN}$, onde a média foi de 50,9 e 54,1\% respectivamente. Já para a relação de peso e potência de $60 \mathrm{~N} \mathrm{~kW}^{-1}$ não houve diferença entre médias nas cargas de 25 a 30 e 35 a $40 \mathrm{kN}$.
\end{abstract}

Palavras-chave: Eficiência trativa. Carro dinamométrico. Ensaio de tratores.

\begin{abstract}
Testing the tractor in agricultural soil is one way of getting information, particularly with regard to the development of traction. The yield at the drawbar is often used to compare or evaluate tractors. However, drawbar performance is affected by the conditions of the displacement surface, the gear ratio and the ratio between the weight and power of the tractor. The objective of this study was to evaluate the yield at the drawbar of a tractor at different weight to power ratios, with different loads on the drawbar. The test was conducted at the Lageado Experimental Farm ,belonging to UNESP, on Botucatu Campus in SãoPaulo, by evaluating the following weight to power ratios: 50, 55 and $60 \mathrm{~N} \mathrm{~kW}^{-1}$, where for each of these ratios, three intervals of force on the drawbar were used, as follows: 15 to 20,25 to 30 and 35 to $40 \mathrm{kN}$. It was concluded that the weight to power ratios and the loads studied showed little variability. The weight to power ratios of 50 and $55 \mathrm{~N} \mathrm{~kW}^{-1}$, for loads on the drawbar of 25 to 30 and 35 to $40 \mathrm{kN}$, showed differences between their averages, with the highest yield at the drawbar being obtained for a load of 25 to $30 \mathrm{kN}$, where the average was 50.9 and $54.1 \%$ respectively. As for the weight to power ratio of $60-\mathrm{N} \mathrm{kW}^{-1}$, there was no difference in the averages for loads of 25 to 30 and 35 to $40 \mathrm{kN}$.
\end{abstract}

Key words: Tractive efficiency. Dynamometer car. Tractor testing.

\footnotetext{
* Autor para correspondência

${ }^{1}$ Recebido para publicação em 26/09/2011; aprovado em 20/06/2012

Pesquisa financiada pelo Departamento de Engenharia Agrícola da Universidade Federal do Ceará

${ }^{2}$ Departamento de Engenharia Agrícola/DENA, Universidade Federal do Ceará, Bloco804, Campus do Pici, Fortaleza-CE, Brasil, 60.455-760, aiveca@ufc.br, daniel.albiero@gmail.com, fabiohenrique@agronomo.eng.br, rafaela_2708@hotmail.com,igor_m_cordeiro@hotmail.com
} 


\section{INTRODUÇÃO}

O ensaio de trator em solo agrícola é uma das maneiras de obter informações, principalmente no que diz respeito ao seu desenvolvimento de tração (CORDEIRO, 2000). Esses ensaios visam à obtenção de informações sobre o desempenho dos rodados, relacionados com as características da interação com o solo (MIALHE, 1996). Neste contexto, Masiero (2010) afirmou que a análise operacional procura desenvolver técnicas que permitam obter o máximo de rendimento útil de todos os recursos disponíveis, por isso é necessário conhecer a força e a potência disponível na barra de tração.

Monteiro, Lanças e Gabriel Filho (2009) constataram que a adição de peso ao trator obedecendo a critérios de relação entre o peso e a potência, acarreta em melhorias em termos de rendimento operacional.

Salvador, Mion e Benez(2009) ao avaliar a relação de consumo específico de combustível concluíram que os menores valores de consumo específico de combustível significam a otimização do desempenho do motor, da eficiência trativa e da adequação do equipamento a fonte de potência. Smerda e Cupera (2010), afirmam que a redução na pressão de inflação e o uso do tipo adequado dos pneus podem melhorar as características de força de tração e consequentemente diminuírem o consumo de combustível.

Masiero (2010) observou que em diferentes superfícies de solo, os valores médios de rendimento máximo na barra de tração variaram em função do modelo, potência do trator e da relação entre o peso e a potência do motor. Avaliando as condições da superfície do solo, Gabriel Filho et al. (2010), concluíram que esta interferiu na capacidade do trator em desenvolver a tração, pois a maioria dos parâmetros relacionados com o desempenho apresentou variações estatisticamente significativas ao longo dos ensaios. Ao se dividir os valores obtidos de potência na barra de tração pelos valores de potência na tomada de potência, obtêm - se os diversos rendimentos da transmissão, de posse dessas informações, têm-se os índices que representam a eficiência energética dos tratores (SILVEIRA; SIERRA, 2010).

Segundo Monteiro, Lanças e Guerra (2011), para diferentes modelos de tratores, os valores médios de rendimento na barra de tração podem variar em função da relação entre o peso e a potência do motor.

Segundo Zoz e Grisso (2003), o principal ponto a ser observado nos tratores agrícolas é o desempenho na barra de tração. A relação entre o peso e a potência dos tratores agrícolas fabricados e comercializados no Brasil variam, basicamente, em função da marca e modelo do trator e da potência do motor. (SCHLOSSER et al., 2005).
Para Alencar, Lopes e Souza (2007) o Controle Estatístico do Processo é uma prática que permite verificar o comprometimento do processo quanto a limites superiores/ inferiores e detectar comportamentos tendenciosos das suas variáveis do processo.

De acordo com Ribeiro e Caten (2011) o CEP é uma ferramenta que pode ser utilizada quando suas amostras possuem distribuição normal; neste enfoque Montgomery (2004) sugere a utilização do gráfico de média móvel exponencialmente ponderada (MMEP) para a análise de processos não normais. Albiero et al. (2012) utilizaram a MMEP com sucesso para avaliar processos não normais em semeadoras, indicando adaptações na metodologia. Paulini, Milan e Salvi (2009) afirmam que é muito complicado determinar todos os fatores que influem nas operações agrícolas, desse modo é preciso o uso de ferramentas de qualidade para garantir o desempenho adequado do processo em função dos fatores críticos.

De acordo Albiero (2010), em geral o controle estatístico do processo (CEP) considera que suas amostras apresentam distribuição normal, porém nem sempre isso ocorre, pois existem limitações graves com relação à normalidade. $\mathrm{O}$ presente trabalho teve por objetivo avaliar o rendimento na barra de tração de um trator agrícola com diferentes relações de peso e potência sobre diferentes regimes de cargas aplicadas na barra de tração.

\section{MATERIAL E MÉTODOS}

O experimento foi conduzido na Fazenda Experimental Lageado, pertencente à Faculdade de Ciências Agronômicas da Universidade Estadual Paulista - UNESP, Campus de Botucatu, com as seguintes coordenadas geográficas de $22^{\circ} 51^{\prime} \mathrm{S}, 48^{\circ} 25^{\prime} \mathrm{W}$ e altitude de $770 \mathrm{~m}$.

A caracterização do solo da área experimental foi descrito conforme Monteiro, Lanças e Guerra (2011). O presente trabalho foi desenvolvido em uma pista de solo firme com $400 \mathrm{~m}$ de comprimento e largura de $20 \mathrm{~m}$, o que totaliza uma área de $8.000 \mathrm{~m}^{2}$.

Utilizou-se a UMEB - Unidade Móvel de Ensaio na Barra de Tração, que simula forças na barra de tração do trator, conforme descrito por Monteiro et al. (2007) e Gabriel Filho et al. (2008).

Para o ensaio utilizou-se um trator $4 \times 2$ TDA da marca John Deere modelo $6600 \mathrm{de} 88 \mathrm{~kW}(121 \mathrm{cv})$ de potência no motor, a 2.100 rotações por minuto. A marcha de trabalho utilizada no ensaio foi a $\mathrm{C} 1$, que corresponde à velocidade teórica sem carga de $7,0 \mathrm{~km} \mathrm{~h}^{-1}$, conforme consta no painel do trator; o ensaio foi realizado com a 
tração dianteira auxiliar ligada. A pressão de inflação para os pneus diagonais, foi de $124,02 \mathrm{kPa}$ (18 psi) nos rodados dianteiros e de $137,8 \mathrm{kPa}$ (20 psi) nos rodados traseiros, conforme recomendação do fabricante dos pneus. O trator foi equipado com um conjunto de pneus diagonais com dimensões de 14.9-26 para os rodados dianteiros e 18.4-30 para os rodados traseiros do trator.

As relações peso/potência utilizadas no ensaio foram de 50; 55 e $60 \mathrm{~N} \mathrm{~kW}^{-1}$, onde para cada uma dessas relações utilizou-se três intervalos de força na barra de tração variando da seguinte forma: 15 a 20; 25 a 30 e 35 a $40 \mathrm{kN}$. A colocação e distribuição de lastro no trator foram realizadas conforme descrito por Monteiro e Arbex (2009).

Para aquisição e acompanhamento dos sinais obtidos pelos sensores instalados no sistema de alimentação de combustível e na barra de tração utilizouse um controlador lógico programável (PLC). Para medição do consumo de combustível foram utilizados dois fluxômetros volumétricos M-III, da FLOWMATE fabricado pela OVAL Corporation do Japão e distribuído no Brasil pela K\&K do Brasil, modelo LSN41L8-M2, vazão de $1 \mathrm{~mL} /$ pulso. Os valores da força na barra de tração foram obtidos através de uma célula de carga marca SODMEX, modelo N400, com sensibilidade de 2,16 mV/ $\mathrm{V}$ e escala nominal de $100 \mathrm{kN}$.

O cálculo do rendimento na barra de tração foi realizado utilizando a Equação 1, e os valores de rendimento na barra de tração foram determinados também através da Equação1.

$$
R b t=\frac{P b}{P m} \times 100
$$

Onde: $\mathrm{Rbt}=$ rendimento na barra de tração $(\%) ; \mathrm{Pb}=$ potência na barra de tração $(\mathrm{kW}) ; \mathrm{Pm}=$ potência no motor

Ao se aplicar os testes estatísticos foi necessário testar a normalidade dos dados estudados. Os testes aplicados a este estudo foram os de Anderson-Darling, assimetria e curtose. No caso de não normalidade dos dados foi utilizada no trabalho a Média Móvel Exponencialmente Ponderada (MMEP), conforme o modelo proposto por Montgomery (2004), que é praticamente um teste não-paramétrico, pois não depende de normalidade da distribuição dos dados. Segundo o mesmo, para usar os gráficos de MMEP existe a possibilidade de os dados do processo não seguirem distribuição normal. $\mathrm{O}$ autor define a MMEP como sendo:

$$
z i=\lambda \cdot \sum_{0}^{i-1}(1-\lambda) \cdot x_{i-j}+(1-\lambda)^{i} \cdot z o
$$

Onde: zi é o valor da média móvel ponderada; zo é a média alvo do processo; xi é o valor da característica medida; $\lambda$ é o peso considerado para a média, se refere à sensibilidade em captar pequenas mudanças na média.

Para o a geração dos gráficos de MMEP utilizouse o peso considerado para a média que se refere à sensibilidade em captar pequenas mudanças na média.

Os limites de controle do gráfico MMEP são dados pelas seguintes equações:

$$
L S C=\mu+L . \sigma \cdot \sqrt{\frac{\lambda}{(2-\lambda)} \cdot\left[1-(1-\lambda)^{2 i}\right]}
$$

$$
L I C=\mu-L \cdot \sigma \cdot \sqrt{\frac{\lambda}{(2-\lambda)} \cdot\left[1-(1-\lambda)^{2 i}\right]}
$$

Em que: LSC é o limite superior de controle; LIC é o limite inferior de controle; $\mu$ é a média do processo; $\mathrm{L}$ é a largura da faixa entre a média e o limite; $\sigma$ é o desvio padrão da amostra.

\section{RESULTADOS E DISCUSSÃO}

Os dados apresentaram-se sem normalidade, portanto optou-se pelo uso irrestrito da MMEP como fator de avaliação. Observa-se na Figura 1, os gráficos de controle que mostram que em todas as relações peso/ potência avaliada, a carga 15 a $20 \mathrm{kN}$ apresentaram alguns pontos fora do limite de controle, neste caso segundo Montgomery (2004) o processo é considerado instável. Verifica-se que todas as relações peso/potência e todas as cargas estudadas apresentaram pouca variabilidade. É possível observar que na relação peso/potência de 50 e $55 \mathrm{~N} \mathrm{~kW}^{-1}$, houve diferença entre médias para as cargas de 25 a 30 e 35 a $40 \mathrm{kN}$, sendo que obteve-se o maior rendimento da barra de tração para a carga de 25 a $30 \mathrm{kN}$, onde a média foi de 50,9 e 54,1\% respectivamente, corroborando com os resultados obtidos por Monteiro, Lanças e Gabriel Filho(2009), que a adição de peso ao trator obedecendo a critérios de relação entre o peso e a potência, acarreta em melhorias em termos de eficiência energética do trator.

Já para a relação peso/potência de $60 \mathrm{~N} \mathrm{~kW}^{-1}$ não houve diferença entre média nas cargas de 25 a 30 e 35 a $40 \mathrm{kN}$, respectivamente.

Analisando as médias de rendimento na barra de tração podemos perceber que com o aumento da relação entre o peso e a potência aumenta a eficiência do trator em realizar tração obtendo-se assim, os maiores rendimento na barra de tração, considerando o rendimento na barra 
Figura 1 - Graficos da média movel exponencialmente ponderada do rendimento na barra de tração de um trator agricola
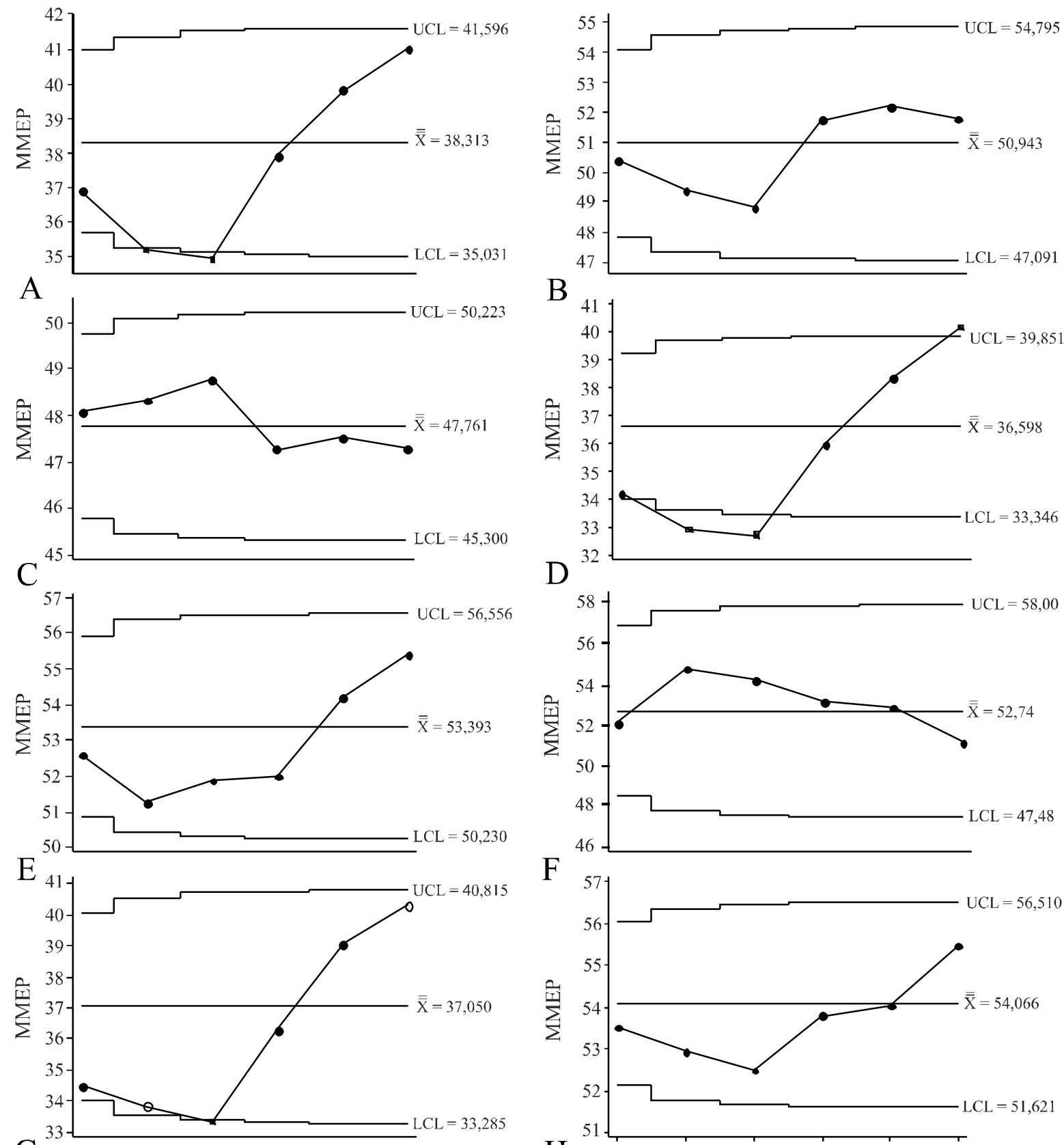

G

F
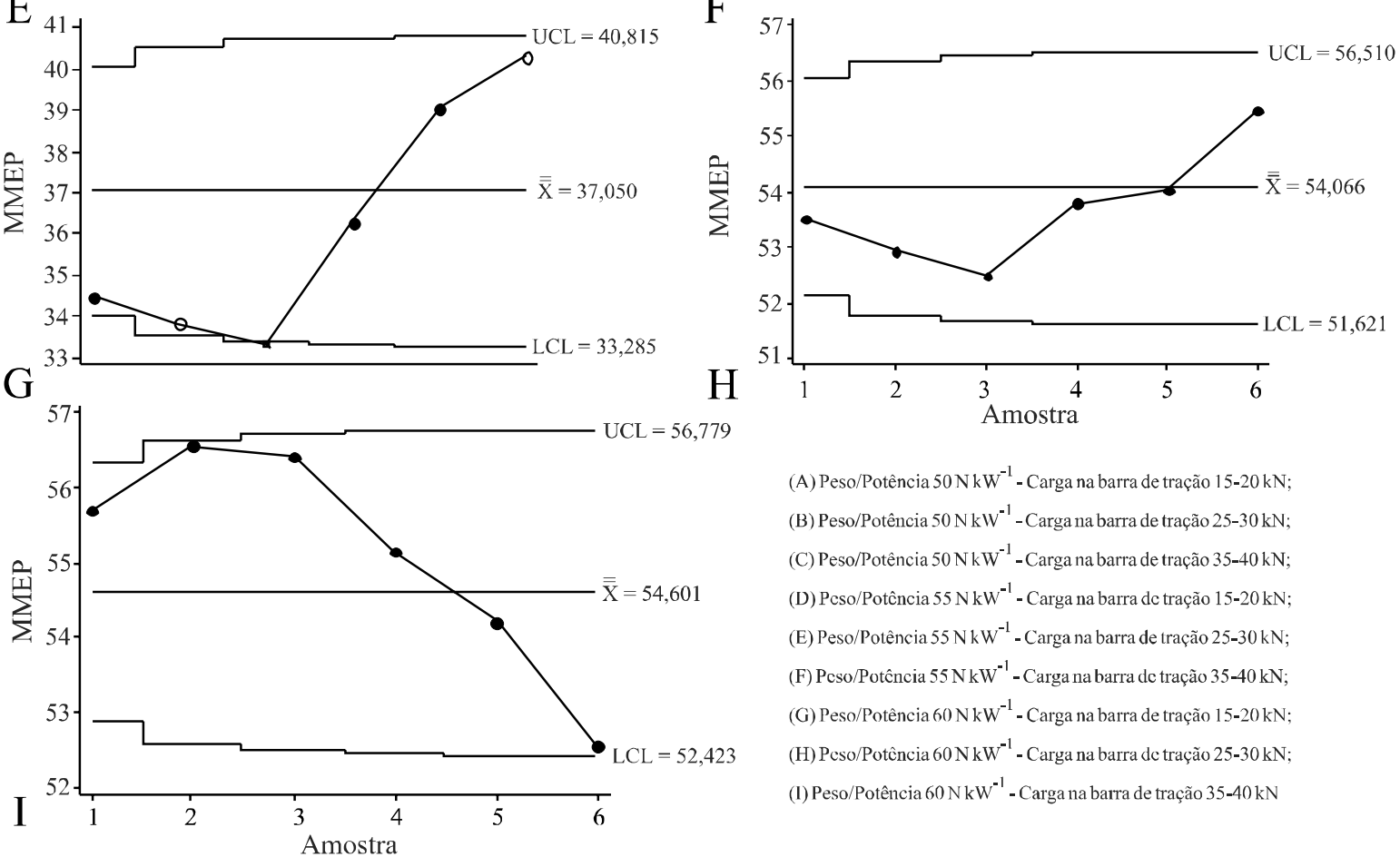

(A) Peso/Potência $50 \mathrm{~N} \mathrm{~kW}^{-1}$ - Carga na barra de tração $15-20 \mathrm{kN}$; (B) Peso/Potência $50 \mathrm{NkW}^{-1}$ - Carga na barra de tração $25-30 \mathrm{kN}$; (C) Peso/Potência $50 \mathrm{NkW}^{-1}$ - Carga na barra de tração $35-40 \mathrm{kN}$; (D) Peso/Potência $55 \mathrm{~N} \mathrm{~kW}^{-1}$ - Carga na barra de tração $15-20 \mathrm{kN}$; (E) Peso/Potência $55 \mathrm{~N} \mathrm{~kW}^{-1}$ - Carga na barra de tração $25-30 \mathrm{kN}$; (F) Peso/Potência $55 \mathrm{~N} \mathrm{~kW}^{-1}$ - Carga na barra de tração $35-40 \mathrm{kN}$; (G) Peso/Potência $60 \mathrm{~N} \mathrm{~kW}^{-1}$ - Carga na barra de tração $15-20 \mathrm{kN}$; (H) Peso/Potência $60 \mathrm{~N} \mathrm{~kW}^{-1}$ - Carga na barra de tração $25-30 \mathrm{kN}$; (I) Peso/Potência $60 \mathrm{~N} \mathrm{~kW}^{-1}$ - Carga na barra de tração $35-40 \mathrm{kN}$ 
de tração como uma medida segundo o qual o sistema rodado-solo transforma o torque do motor em tração na barra. Resultados semelhantes foram obtidos por Masiero (2010), afirmou o autor que os valores médios de rendimento na barra de tração variaram em função da relação entre o peso e a potência do motor.

Com o aumento da carga na barra de tração podemos perceber que para as condições avaliadas a faixa de carga que melhor se adéqua ao sistema é a de 25 a $30 \mathrm{kN}$ onde se tem as melhores médias para o rendimento na barra de tração, aumentando as cargas para $40 \mathrm{kN}$ nota-se uma queda no rendimento na barra de tração principalmente para as relações peso/potência de 50 e $55 \mathrm{~N} \mathrm{~kW}^{-1}$. Monteiro, Lanças e Guerra (2011), afirmaram que valores médios de rendimento na barra de tração podem variar em função da relação entre o peso e a potência do motor, isso ocorre devido ao excesso de carga na barra de tração que influencia na capacidade máxima de tração que um trator pode desenvolver sob as condições do experimento, isso ocorre em função da magnitude do torque que o conjunto motor-transmissão é capaz de aplicar às rodas motrizes, do limite do potencial do solo agrícola para o desenvolvimento de força de tração e da resistência externa ao rolamento.

\section{CONCLUSÕES}

1. Entre as relações de peso e potência, o maior rendimento na barra de tração do conjunto moto mecanizado foi obtido para a relação peso potencia de $60 \mathrm{~N} \mathrm{~kW}^{-1}$, nas cargas aplicadas na barra de tração com amplitude de 25 a $40 \mathrm{kN}$;

2. Os melhores rendimentos da barra de tração do trator foram obtidos quando se aplicou cargas na barra de tração de 25 a $30 \mathrm{kN}$ nas relações entre o peso e a potência de 50 e $55 \mathrm{~N} \mathrm{~kW}^{-1}$, mostrando ser a amplitude de carga mais adequada ao conjunto avaliado.

\section{REFERÊNCIAS}

ALBIERO, D. Desenvolvimento e avaliação de máquina multifuncional conservacionista para a agricultura familiar. 2010. 244 f. Tese (Dourado em Engenharia Agrícola) Universidade Estadual de Campinas, São Paulo, 2010.

ALBIERO, D. et al. Avaliação da distribuição de sementes por uma semeadora de anel interno rotativo utilizando média móvel exponencial. Revista Ciência Agronômica, v. 43, n. 1, p. 86-95, 2012.

ALENCAR, J. R. B.; LOPES, C. E.; SOUZA JUNIOR. M. B. Controle Estatístico de Processo Multivariado: aplicação ao monitoramento da produção de comprimidos de captopril. Revista Brasileira de Farmácia, v. 88, n. 4, p. 200-205, 2007.

CORDEIRO, M. A. L. Desempenho de um trator agrícola em função do pneu, da lastragem e da velocidade de deslocamento. 2000. 153 f. Tese (Doutorado em Agronomia) Universidade Estadual Paulista, São Paulo, 2000.

EMPRESA BRASILEIRA DE PESQUISA AGROPECUÁRIA. Centro Nacional de Pesquisa de Solos. Sistema Brasileiro de classificação de solos. Rio de Janeiro.1999. 412 p.

GABRIEL FILHO, A. et al. UMEB - Unidade móvel para ensaio da barra de tração. Engenharia Agrícola, v. 28, n. 4, p. 782-789, 2008.

GABRIEL FILHO, A. et al. Influência da altura das garras dos pneus de um trator em área de plantio direto. Revista Brasileira de Engenharia Agrícola e Ambiental, v. 14, n. 10, p. 1123-1128, 2010.

MASIERO, F. C. Determinação do rendimento na barra de tração de tratores agrícolas com tração dianteira auxiliar (4X2 TDA). 2010. 79 f. Dissertação (Mestrado em Agronomia/ Energia na Agricultura) - Faculdade de Ciências Agronômicas, Universidade Estadual Paulista, Botucatu, 2010.

MIALHE, L. G. Máquinas agrícolas: ensaio \& certificação. Piracicaba: Fundação de Estudos Agrários "Luiz de Queiroz", 1996. $722 \mathrm{p}$.

MONTEIRO, L. A. et al. Construção e Avaliação da Unidade Móvel para Ensaio na Barra de Tração. In: CONGRESSO BRASILEIRO DE ENGENHARIA AGRÍCOLA, 36., 2007, Bonito. Anais... Bonito: SBEA, 2007. 1 CD-ROM.

MONTEIRO, L. A.; LANÇAS, K. P.; GABRIEL FILHO, A. Desempenho de um trator agrícola em função do tipo construtivo do pneu e da lastragem líquida em três velocidades de deslocamento na pista com superfície firme. Energia na Agricultura, v. 24, n. 1, p. 68-84, 2009.

MONTEIRO, L. A.; LANÇAS, K. P.; GUERRA S. P. S. Desempenho de um trator agrícola equipado com pneus radiais e diagonais com três níveis de lastros líquidos. Engenharia Agrícola, v. 31, n. 3, p. 551-560, 2011.

MONTEIRO, L. A.; ARBEX, P. R. Operação com tratores agrícolas. Botucatu: Ed. dos Autores, 2009.76 p.

MONTGOMERY, D. C. Introdução ao controle estatístico da qualidade. 4. ed. Rio de Janeiro: LTC, 2004.

PAULINI, D. G.; MILAN, M.; SALVI, J. V. Qualidade total. Cultivar Máquina, ${ }^{0}$ 85, Pág 23 a 27, 2009.

RIBEIRO, J. L. D.; CATEN, S. T. Controle estatístico do processo: cartas de controle para variáveis,cartas de controle para atributos, função de perda quadrática, análise de sistemas de medição. Porto Alegre: FEENG/UFRGS, 2011. 156 p. (Série Monográfica Qualidade).

SALVADOR, N.; MION, R. L.; BENEZ, S. H. Consumo de combustível em diferentes sistemas de preparo periódico realizados antes e depois da operação de subsolagem. Ciência Agrotécnica, v. 33, n. 03, p. 870-874, 2009. 
SCHLOSSER, J. F. et al. Análise comparativa do peso específico dos tratores agrícolas fabricados no Brasil e seus efeitos sobre a seleção e uso. Ciência Rural, v. 35, n. 1, p. 92-97, 2005.

SILVEIRA, G. M.; SIERRA, J. G. Eficiência energética de tratores agrícolas fabricados no Brasil. Revista Brasileira de Engenharia Agrícola e Ambiental, v. 14, n. 4, p. 418-424, 2010.
SMERDA, T.; CUPERA, J. Tire inflation and its influence on drawbar characteristics and performance - Energetic indicators of a tractor set. Journal of Terramechanics, v. 47, n. 6, p. 395400, 2010.

ZOZ, F.; GRISSO, R. D. Traction and tractor performance. St. Joseph: ASAE, 2003.5 p. (Tractor desing, 27). 